\title{
ASEGURAMIENTO DE LA CALIDAD DE LA EDUCACIÓN SUPERIOR EN AMÉRICA LATINA: ¿VAMOS POR EL CAMINO CORRECTO?
}

\author{
Mónica Pérez*
}




\section{RESUMEN}

Los objetivos del presente artículo son realizar un balance sobre el desarrollo de las experiencias de aseguramiento de la calidad de la educación superior en América Latina y contribuir a la reflexión sobre los escenarios futuros que enfrentan dichas experiencias.

En la primera parte se exponen los factores más comunes que caracterizan a los sistemas educativos latinoamericanos y que inciden directamente en la calidad de los mismos; en la segunda parte se revisan los sistemas de evaluación de la calidad de América Latina dentro del contexto descrito y, por último, se exponen ciertas reflexiones generadas como consecuencia de la realidad observada.

\section{ABSTRACT}

The purpose of this article is to prepare a balance on the development of quality assurance experiences in higher education in Latin America and contribute to reflect on the future scenarios that these experiences will face.

The first part of the paper presents the most common factors that characterize Latin American educational systems and that have a direct effect on their quality; the second part reviews quality assessment systems in Latin America in the context described and, lastly, the paper ends by providing some reflections which emerge as a result of the reality observed. 


\section{ASEGURAMIENTO DE LA CALIDAD DE LA EDUCACIÓN SUPERIOR EN AMÉRICA LATINA: ¿VAMOS POR EL CAMINO CORRECTO?}

Introducción

El presente documento se funda en un proyecto de investigación, realizado por la autora, en el que se examinan los sistemas de aseguramiento de calidad de la Educación Superior en América Latina.

Los gobiernos latinoamericanos están entendiendo que la falta de adecuación de sus sistemas de educación superior a las nuevas exigencias de un mundo globalizado significa quedarse atrás en la carrera del desarrollo, por lo que ya no se discute la importancia de implementar mecanismos destinados a asegurar la calidad de dicha educación, sino que el foco de atención está centrado en la eficacia de dichos sistemas. Como señala el Banco Mundial en su informe Constructing Knowledge Societies: New Challenges for Tertiary Education (2002, p. XIX), "los países en desarrollo y transición afrontan el riesgo de ser aún más marginados de la economía mundial, debido a que sus sistemas de educación superior no están adecuadamente preparados para capitalizar sobre la base de la creación y uso del conocimiento". En consecuencia, ya no basta con la instalación de políticas de aseguramiento de la calidad, sino que su eficacia resulta clave en la sociedad del conocimiento.

Es muy importante conocer la forma en que los distintos países enfrentan sus propias realidades, ya que si el desarrollo de América Latina depende en gran parte de la calidad de su educación superior, cabe preguntarse si sus gobiernos están actuando con eficacia frente a 
este desafío, con políticas a largo plazo; o, más bien, obedecen a otros motivos demagógicos y cortoplacistas que no están necesariamente vinculados a un mejoramiento o aseguramiento de la calidad y sin una real conciencia de la importancia de este tema.

Las pregunta que tratamos de responder es: ¿de qué forma están enfrentando los distintos países este desafío?

No pretendemos realizar aquí un análisis detallado de países individualmente considerados, sino sólo abordar problemas comunes o de carácter general que los afectan.

Los objetivos del presente artículo son realizar un balance sobre el desarrollo de experiencias de aseguramiento de la calidad en América Latina y contribuir a la reflexión sobre los escenarios futuros que enfrentan dichas experiencias.

En la primera parte expondré los factores más comunes que caracterizan a los sistemas educativos latinoamericanos y que inciden directamente en la calidad de los mismos; en la segunda, realizaré una revisión de los sistemas de aseguramiento de la calidad de América Latina dentro del contexto descrito y en la tercera parte plantearé algunas reflexiones producto de los análisis anteriores.

Descripción general del escenario latinoamericano

Existen varios factores comunes que inciden directamente en el desarrollo de los sistemas de aseguramiento de la calidad en Latinoamérica. En efecto, la expansión de la matrícula, el aumento del número de instituciones de educación superior, la disminución del gasto público en educación y el nuevo rol que deben asumir sus distintos actores, han traído como consecuencia una creciente preocupación de los gobiernos latinoamericanos por la calidad, lo que se ha traducido en el desarrollo de políticas gubernamentales orientadas a cautelar la fe pública y a informar y proteger al usuario.

La expansión de la matrícula y, por consiguiente, del número de instituciones de educación superior, obedeció más a oportunidades de mercado que a una expansión planificada y controlada. En efecto, la mayoría de los países latinoamericanos optó por restringir el gasto 
público y liberalizar el mercado de la educación superior en condiciones poco favorables a la calidad, dado que en muchos de esos países no existía una cultura de rendición de cuentas o evaluación de la calidad, sino que, muy por el contrario, el modelo de universidad existente en ellos tendía hacia modelos bastante autocomplacientes, basados en una autonomía mal entendida, fuertemente politizados y con una relación con el Estado basada en la negociación y el conflicto. En consecuencia, la apertura del mercado de la educación superior vino a complicar mucho más el panorama de este tipo de educación en Latinoamérica, lo que se tradujo en un fenómeno de masificación, privatización y estratificación y, en definitiva, en una pérdida de la calidad, eficiencia y eficacia. Los sistemas de educación superior se volvieron caóticos, con instituciones relativamente pequeñas que aparecían en forma descontrolada, duplicando la oferta ya existente de carreras, volviéndose ineficientes, poco articulados y compitiendo por la captación de alumnos en lugar de por la excelencia académica, todo ello favorecido, además, por la falta de transparencia e información del sistema.

Los sistemas nacionales de educación superior latinoamericanos se caracterizan por ser bastante heterogéneos. Coexisten en un mismo sistema establecimientos nuevos con antiguos, universitarios y no universitarios, públicos y privados, con y sin investigación; todos ellos con distintas orientaciones y niveles de calidad. Si a lo anterior le añadimos la internacionalización de la educación superior, obtenemos sistemas todavía más heterogéneos en los que conviven instituciones provenientes del sector público y del sector privado nacional, y del sector privado extranjero, operando estas últimas a través de franquicias o alianzas estratégicas, entre otros mecanismos.

La masificación de los sistemas de educación superior, junto con la heterogeneidad de las instituciones que los conforman, dificulta la tarea de los estados en orden a impulsar políticas públicas homogéneas destinadas a desarrollar dichos sistemas como un todo. Dicha heterogeneidad se ha traducido en una fragmentación legislativa, es decir, una proliferación de leyes destinadas a los distintos tipos de instituciones. Este fenómeno constituye un obstáculo en la tarea de coordinación y articulación de la educación superior, propiciando sistemas pocos articulados, complejos y de crecimiento inorgánico. 
Por otra parte, el crecimiento de las instituciones privadas de educación superior significó un aumento de matrícula en términos generales. Sin embargo, ello no redundó necesariamente en una mayor cantidad de alumnos matriculados en instituciones privadas, sino que, si observamos la conformación de los distintos sistemas educativos según matrícula, proporcionalmente esta es mayor en las universidades públicas que en las privadas, fenómeno que es indicativo de su importancia en Latinoamérica. Las universidades públicas en la región son instituciones con bastante poder de negociación dentro de los sistemas, dotadas de una gran autonomía, fuertemente politizadas, tradicionalmente monopólicas, con asignaciones presupuestarias aseguradas en forma automática, sin tener como contrapartida, algún tipo de rendición de cuentas y sin responder públicamente por su desempeño. Es más, existen países en los que la dirección y planificación de la educación superior está en manos de dichas instituciones, sin que los ministerios de educación tengan injerencia alguna.

Finalmente, casi todos los países han sufrido una reducción de sus presupuestos destinados a la educación superior, lo que ha presionado a los distintos estados e instituciones de este tipo de educación a preocuparse de que los recursos sean invertidos en forma cada vez más eficiente. El Estado ya no se limita a ser mero suministrador de los recursos asignados a las instituciones públicas (en muchos países dicha asignación está fijada constitucionalmente), sino que exige eficiencia en la utilización de los mismos, lo que obliga a las instituciones a dar cuenta de su uso. Por otro lado, el ciudadano exige, a su vez, que den cuenta de su desempeño a la sociedad, por lo que éstas sienten la presión de estar revisando constantemente su misión, visión y vinculación con la realidad de los países.

Los sistemas latinoamericanos de aseguramiento de la calidad de la educación superior

Existen diversos factores que están detrás de las iniciativas evaluativas de la educación superior en América Latina. Tyler y Bernasconi (1999) señalan algunos de carácter general, como la expansión de la matrícula, la creación no regulada de instituciones y la disminución en las universidades del gasto público por estudiante, factores que se han traducido en una mayor preocupación de los gobiernos por la calidad de las 
universidades, ya sea con fines de proteger e informar al consumidor, dar respuesta pública a una demanda de aseguramiento de la calidad, permitir una mayor movilidad a los estudiantes como consecuencia de los procesos de integración o, simplemente, para no quedar marginados de la nueva economía mundial y salir del subdesarrollo.

Sin embargo, es necesario preguntarse si la preocupación por la calidad de la educación superior es una preocupación de fondo o meramente formal. ¿Es suficiente e indicativo de que estamos en el camino correcto? Es evidente que existe una mayor preocupación en los distintos gobiernos latinoamericanos y en las distintas comunidades académicas. Sin embargo, ¿basta con la instalación de dichos sistemas para mejorar la calidad de la educación superior? $\mathrm{O}$ ¿existen una serie de factores u obstáculos al interior de cada sistema que están impidiendo su desarrollo eficaz?

La respuesta a lo anterior es especialmente relevante si tenemos en cuenta que uno de los factores que incide directamente en el desarrollo de un país es la calidad de su educación. Es necesario entonces revisar el estado del arte de los sistemas de aseguramiento de la calidad de la educación superior en América Latina, para conocer la forma en que los distintos países los están implementando o desarrollando. De esta forma podemos obtener una visión general acerca de su estado actual y determinar, en una primera aproximación, los obstáculos que impiden un mayor grado de desarrollo de los mismos.

La mayoría de los países latinoamericanos instaló sus sistemas de aseguramiento de la calidad en la segunda mitad de la década de los 90, a excepción de unos pocos países que ya tenían algún tipo de sistema de regulación o control instalado con anterioridad (Brasil, Chile, Colombia, Costa Rica, Cuba, México y República Dominicana).

Sin embargo, los sistemas de más antigua data obedecían en un comienzo a finalidades de control (muchas veces de tipo administrativo), más en un intento por frenar la expansión del sistema que a finalidades de mejoramiento de la calidad de sus instituciones. En efecto, se trata de países en donde el número de instituciones creció en forma importante, en casi todos es mayor el número de privadas que de públicas y hay un predominio de matrícula privada sobre 
matrícula pública. En estos países se implementaron ciertos mecanismos de control destinados a supervisar, vigilar, aprobar o rechazar los diferentes programas, así como a ejercer labores de control administrativo. Ejemplo de ello es el Instituto Colombiano para el Fomento de la Educación Superior (ICFES), el Consejo Nacional de Educación Superior Privada (CONESUP), de Costa Rica, la Comisión Nacional para la Evaluación de la Educación Superior (CONAEVA), de México, y el Consejo Nacional de Educación Superior (CONES), de República Dominicana.

Sin embargo, podemos observar que muchos de los procesos ya establecidos en este grupo de países se encuentran en proceso de revisión o recientemente acaban de crear nuevos sistemas. Este es el caso de Costa Rica en que, mediante la Ley 8.256 de mayo de 2002, se establece el Sistema Nacional de Acreditación de la Educación Superior (SINAES). En Chile existe un proyecto de ley que establece un Sistema Nacional de Aseguramiento de la Calidad de la Educación Superior, y en México la Asociación Nacional de Universidades e Instituciones de Educación Superior (ANUIES) inscribió el acuerdo de impulsar el Sistema de Evaluación y Acreditación de la Educación Superior, creando una comisión encargada de elaborar la propuesta para consolidar el Sistema Nacional de Evaluación y Acreditación.

La mayoría de los sistemas tiene como finalidad el mejoramiento o promoción de la calidad de la educación superior y la acreditación de las carreras o programas que cumplan con unos estándares o criterios previamente definidos, en combinación con el respectivo proyecto institucional. Los procedimientos son bastante similares, $\mathrm{e}$ incluyen la autoevaluación realizada por la propia institución, la visita a la institución realizada por pares evaluadores externos y la evaluación final.

Sin embargo, existen varios factores que, a nuestro juicio, afectan la eficacia de los sistemas instalados. En efecto, en varios de los países analizados no existen sistemas de información desarrollados y, en algunos casos, los resultados de los procesos son confidenciales y no conducen a sanción o incentivo alguno, por lo que las declaraciones de promoción de la calidad pudieran no tener la eficacia deseada. Lo anterior resulta particularmente relevante si en el sistema no existe una cierta cultura de calidad previamente instalada y una competencia 
al interior del mismo que incentive a las instituciones de educación superior a pugnar por recursos, estudiantes y calidad, es decir, a esforzarse por ser cada vez más eficientes y optimizar sus recursos. La falta de información no genera competencia entre las instituciones de educación superior, lo que afecta directamente a su eficiencia y a la eficacia del sistema de aseguramiento de la calidad, corriéndose el peligro de orientarlo hacia una cultura del cumplimiento y burocratización, sin que ello se traduzca en un verdadero mejoramiento.

Un segundo grupo de países son aquellos cuyos sistemas de evaluación de la calidad de la educación superior han sido creados en la segunda mitad de la década de los 90 (Argentina, Bolivia, Ecuador, El Salvador, Paraguay y Venezuela).

Se trata de sistemas que, en un comienzo, se caracterizaron por un crecimiento inorgánico y desordenado de sus instituciones, con una gran heterogeneidad y poca articulación entre los distintos niveles educacionales, con un marco legislativo excesivamente fragmentado y una débil regulación o control estatal. Muchos de ellos se caracterizan, además, por su gratuidad y/o el acceso libre.

En muchos de estos países existen universidades definidas por una fuerte autonomía, cogobierno en la gestión, fuerte corporativismo en su interior, endógenas, sin relación con el entorno y carentes de una cultura de evaluación. Por ejemplo, en Bolivia no se ha podido consolidar todavía un sistema de aseguramiento de la calidad único para todas las instituciones, en parte por la fuerte oposición que ejercieron las universidades públicas a su creación.

Dado el fuerte carácter autónomo de las universidades, en varios países las universidades públicas tomaron la iniciativa y crearon sus propios sistemas de aseguramiento de la calidad, anticipándose a cualquier iniciativa gubernamental. Ejemplo de ello es el Sistema Nacional de Evaluación y Acreditación de la Universidad Boliviana (únicamente para las universidades públicas).

Por otra parte, y siempre dentro de este grupo, se puede observar que existe un mayor nivel de consolidación de los sistemas de 
aseguramiento de la calidad en aquellos países en los que el Estado ejerce la coordinación de la educación superior (Argentina, Ecuador y El Salvador), frente a aquellos en los que la coordinación es ejercida por las propias universidades (Bolivia, Paraguay y Venezuela).

En el primer subgrupo, los sistemas de aseguramiento gozan de una mayor antigüedad, grado de desarrollo y experiencia. La mayoría de los sistemas tiene como finalidad el mejoramiento o promoción de la calidad, realizan evaluación institucional y acreditación de carreras o programas, con procedimientos bastante similares y en base a criterios o estándares previamente definidos.

En el segundo subgrupo de países la coordinación es ejercida corporativamente por el Congreso Nacional de Universidades en Bolivia, el Consejo de Universidades en Paraguay y el Consejo Nacional de Universidades en Venezuela, respectivamente. Dichos organismos están encargados de definir la orientación y desarrollo de sus respectivos sistemas universitarios.

Este grupo se caracteriza por poseer sistemas de carácter incipiente y estar en una etapa de desarrollo. En el Caso de Bolivia, existen dos sistemas distintos, uno creado por las universidades públicas integrantes del Sistema de la Universidad Boliviana, cuya finalidad es el mejoramiento de la calidad de sus instituciones, y otro para las universidades privadas, cuya finalidad es la categorización de éstas como simples o plenas. En el caso de Paraguay y Venezuela, sus sistemas tienen un carácter más reciente, ya que en el año 2003 se creó la Agencia Nacional de Evaluación y Acreditación de la Educación Superior, en Paraguay, y en el año 2001 se constituyó el Sistema Nacional de Evaluación y Acreditación de las Universidades Nacionales, en Venezuela.

Llama la atención que el financiamiento de la mayoría de estos sistemas se encuentre en crisis. A ello hay que añadir que muchos de ellos tienen un carácter gratuito y la admisión es libre, por lo que la demanda por recursos es todavía mayor. Por otra parte, muchos de estos países están atravesando serias crisis políticas, económicas y sociales, lo que ha producido una progresiva disminución del presupuesto destinado al financiamiento de la educación superior y un grave deterioro de sus instituciones. 
En consecuencia, podemos señalar que los sistemas de este segundo grupo de países están atravesando por serias dificultades que impiden un adecuado desarrollo y grado de consolidación. La fuerte autonomía de la que gozan muchas de las universidades públicas se ha traducido en un escollo difícil de sortear por los gobiernos de los distintos países y, por otro lado, para desarrollar sistemas de educación superior coherentes y eficientes a largo plazo es necesario el compromiso de los distintos gobiernos de dotarlos de estabilidad financiera, lo que implica, además, una necesidad de que las políticas instaladas sean a largo plazo y tengan cierto grado de continuidad. Lo anterior supone que los gobiernos tengan la capacidad de desarrollar políticas que sean sustentables a largo plazo, porque, de lo contrario, se corre el riesgo de que la educación superior quede sometida a las fluctuaciones del gasto público.

Un tercer grupo de países son aquellos que todavía no poseen sistemas de aseguramiento de la calidad (Guatemala, Honduras, Nicaragua, Panamá, Perú y Uruguay). La coordinación de la educación superior en estos países está en manos, o bien de las únicas universidades públicas existentes en los mismos o, en el caso de aquellos países en los que existe más de una universidad pública, de los organismos que las agrupan.

Respecto de los primeros (Universidad San Carlos de Guatemala, Universidad Nacional de Honduras y Universidad de Panamá), la facultad de dirigir, organizar y desarrollar la educación superior de sus respectivos países es otorgada constitucionalmente y en forma exclusiva a dichas universidades. En los casos de Nicaragua y Perú, los organismos encargados de elaborar la política nacional de la educación superior de estos países son los que agrupan a sus universidades públicas, esto es, el Consejo Nacional de Universidades, en Nicaragua, y la Asamblea Nacional de Rectores, en Perú.

Uruguay es el único país dentro de este grupo en el que el Estado ejerce algún tipo de coordinación de la educación superior a través de la Comisión Coordinadora de Educación, presidida por el Ministerio de Educación y Cultura. Sin embargo, cabe señalar que esta comisión carece de facultades resolutivas y sólo puede formular recomendaciones. 
Este grupo de países no cuentan con un sistema nacional de aseguramiento de la calidad de la educación superior. La mayoría posee algún tipo de mecanismo de control o supervisión de las instituciones de carácter privado y, en la mayoría de los casos, tiene como finalidad autorizar o rechazar el funcionamiento de nuevas instituciones de este tipo en el país, previo cumplimiento de una serie de estándares mínimos fijados con anterioridad.

El hecho de que estos países no cuenten todavía con sistemas nacionales de aseguramiento de la calidad de la educación superior se debe, en gran medida, a la propia estructura de sus instituciones, dado que cuentan con universidades públicas que, históricamente, fueron las únicas en su país, dotadas de gran autonomía y encargadas de dirigir y controlar este nivel de educación. Por otra parte, se observa que los marcos jurídicos que sustentan los sistemas de educación superior de los países analizados constituyen un serio obstáculo para iniciar cualquier tipo de reforma, dado que, en algunos casos, se otorgan constitucionalmente facultades de coordinación a las universidades públicas sobre la educación superior, por lo que cualquier cambio que se quiera introducir sobre esta materia requerirá de un amplio consenso.

A pesar de lo anterior, algunos países ya venían desarrollando algunas actividades evaluativas en el marco del Sistema Centroamericano de Evaluación y Acreditación de la Educación Superior (SICEVAES), lo que ha favorecido que algunos de ellos ya estén desarrollando proyectos de creación de sistemas nacionales de aseguramiento.

\section{Conclusión}

Podemos observar que son pocos los países latinoamericanos que pueden mostrar resultados significativos en sus sistemas de aseguramiento de la calidad de la educación superior. El progresivo deterioro de la calidad de sus instituciones resulta alarmante si a ello se une la incapacidad o falta de voluntad de los gobiernos por superar los distintos factores que impiden la instalación de sistemas en forma eficaz. En muchos de los países analizados se observa, en general, una carencia de políticas para conducir los sistemas, limitándose a cumplir 
con el financiamiento y sin desempeñar un papel más activo en el desarrollo de la educación superior.

Los sistemas de aseguramiento de la calidad de la educación superior en Latinoamérica están en una fase de desarrollo y consolidación, dentro de un contexto político, económico, social y cultural complejo, por lo que las dificultades y obstáculos que deben salvar son grandes y se requieren de políticas públicas a largo plazo para lograr su afianzamiento.

En consecuencia, resulta difícil instaurar sistemas destinados a dar garantías de calidad sin un desarrollo paralelo de capacidades de autoevaluación y autorregulación en las instituciones de educación superior, así como sin el desarrollo de capacidades de generación y análisis de información para la toma de decisiones. De lo contrario, los procesos instalados tienden a burocratizarse, formalizarse y convertirse en procesos de cumplimiento más que en oportunidades de mejora.

Pensamos que la implementación de sistemas de aseguramiento no resulta ser suficiente si no va acompañada de una cultura de la calidad al interior de las instituciones de educación superior y de un compromiso serio por parte de los gobiernos por promoverla y desarrollarla. De lo contrario, los sistemas se quedarán estancados y entrampados ante la imposibilidad de cambiar las culturas institucionales o de superar las prácticas demagógicas o políticas cortoplacistas, que obedecen a motivos muy diferentes que los de promover una verdadera cultura de excelencia al interior del sistema. 


\section{REFERENCIAS BiBLIOGRÁFICAS}

Amtmann, C, "Nuevas demandas y nuevas respuestas de la universidad. ¿Una nueva crisis?", en: CINDA 2003.

Banco Mundial, Constructing Knowledge Societies: New Challenges for Tertiary Education, Banco Mundial, Washington, D.C., 2002.

Brunner, J.J., Educación Superior en América Latina: cambios y desafios ( $1^{a}$ ed.), Fondo de Cultura Económica, Santiago de Chile, 1990.

Brunner, J.J, "Nuevas demandas y sus consecuencias para la educación superior en América Latina", en: CINDA 2003.

CINDA, Políticas públicas, demandas sociales y gestión del conocimiento ( $1^{\text {a }}$ ed.), Centro Interuniversitario de Desarrollo, Santiago de Chile, 2003.

Conferencia Regional sobre la Educación Superior en América Latina y el Caribe. La Habana, noviembre de 1996.

De Moura Castro, C. y Levy, D., La educación superior en América Latina y el Caribe, Documento de estrategia, Banco Interamericano de Desarrollo, Washington D.C., 1997.

El-Khawas, E., Depietro-Jurand, R. y Hola-Nielsen L., El control de la calidad en la educación superior: avances recientes y dificultades por superar, 1998 [Documento en Internet] Disponible en http: // wwwl.worldbank.org/education/tertiary/documents/ElainSpa3.pdf

Harvey, L., Evaluating the evaluators, Ponencia inicial de la V Conferencia Bienal de la Red de Agencias de Aseguramiento de la Calidad de la Educación Superior (INQAAHE), Santiago de Chile, mayo 1999.

Middlehurst, R. y Woodhouse, D., "Coherent Systems for External Quality Assurance", on Quality in Higher Education 1995, 1 (3), 257-268. 
Rama, C., "Las nuevas demandas de Educación Superior en América Latina", en: CINDA 2003.

The Task Force on Higher Education, Higher Education in Developing Countries: Peril and Promise, 2000 [Website] Available at http:// www.tfhe.net/

Tyler, L.A. y Bernasconi, A., Evaluación de la Educación Superior en América Latina: Tres Órdenes de Magnitud; Development Discusión Paper $N^{\circ} 700,1999$. [Documento en Internet] Disponible en http:// www.incae.ac.cr/ES/clacds/investigacion/pdf/hiid700-cen1201.pdf

Winkler, D., La Educación Superior en América Latina. Cuestiones sobre Eficiencia y Equidad, (Documentos para discusión del Banco Mundial; 77S), Banco Mundial, 1994. 